\title{
Slowing Influenza Virus Evolution: A Role for Multiple Synergistic Antiviral Specificities in Vaccination Strategies
}

\author{
Bernard A.P. Lafont and Jack R. Bennink
}

Keywords: influenza virus, virus evolution, universal vaccines, immune specificity, polyclonal responses

\begin{abstract}
$\mathbf{A}^{5}$ S WE CONSIDERED THE TOPIC of our contribution to this special issue honoring Peter Doherty, we recalled that the central concept of Peter and Rolf Zinkernagel's Nobel award winning findings involved $\mathrm{T}$ cell specificity. Essentially, what do $\mathrm{T}$ cells recognize. The Doherty and Zinkernagel discovery in 1974 of the major histocompatibility complex restriction of $\mathrm{T}$ cells galvanized the field's focus on the $\mathrm{T}$ cell receptor and $\mathrm{T}$ cell antigen specificity (35). Similarly, for B cells Paul Ehrlich had proposed years earlier in his 1908 Nobel lecture (8) that the antitoxins (i.e., antibodies) described by von Behring and Kitasao (29) were preformed antibody receptors that specifically bound antigen. Specificity is key to all adaptive immune responses, and the basis for protective immunity induced by most vaccines typically involves specific antibodies. The specificity of B cell responses can further be critically important clinically as differences in antibody specificity can result in either protection against or enhancement of disease as illustrated in the case of dengue virus infection (23). Currently, major efforts are underway to develop a universal influenza virus vaccine that would provide broadly protective immunity for all influenza A viruses (IAVs). In this brief piece, we suggest that the search for a universal influenza virus vaccination strategy must consider the inclusion of multiple universal target specificities to slow the risk of virus evolution and prevent viral escape.

IAVs cause seasonal outbreaks worldwide and occasionally severe pandemics that are a significant burden in morbidity, mortality, and economic loss. The continuous evolution of IAV through mutations and reassortments provide the overall mechanisms to explain why previous exposure to IAV does not confer permanent protection against IAV infection. The key molecules targeted by B cell immunity are the two ma-
\end{abstract}

jor IAV surface glycoproteins, hemagglutinin (HA) and neuraminidase (NA). For most pandemics, zoonotic IAVs with viral glycoproteins previously uncirculated in humans gain the ability to replicate and efficiently transmit to humans. For seasonal IAV epidemics, progressive antigenic drift, especially around the sialic acid binding site of the HA protein, generates new viral variants with amino acid changes that alter antigenic epitopes and preclude optimal recognition by pre-existing immunity. This antigenic evolution leads to the complete replacement of older strains with new viruses circulating through the human population.

Due to the antigenic evolution of influenza viruses, influenza vaccine compositions are examined every year and adjusted as needed. Predictions of the best guess matched strains have improved over recent years thanks to the extended usage of viral sequencing data. However, it has become evident that additional factors, such as antigenic changes in the HA generated during the production of $\mathrm{H} 3 \mathrm{~N} 2$ vaccines in eggs, complicate further the attempt of matching the vaccines with circulating strains and result in decreased hemagglutination titers against these viruses (24). These limitations, as well as the more pressing fear of the emergence of a potential pandemic, particularly from the introduction of highly lethal IAV strains into the human population, have made improving influenza vaccination a public health priority (27).

Creating a universal influenza vaccine has long been a goal of influenza researchers. In the late 70s, CD8+ T cell responses to influenza were discovered to have broadly crossreactive specificities to peptides derived from more conserved internal proteins, creating hope of a more universal T cell-based vaccine (7). Although crossreactive, the modest protective effect and lack of sterilizing immunity provided by $\mathrm{T}$ cells make these responses less than ideal.

Viral Immunology Section, NIAID, NIH, Bethesda, Maryland.

(C) Bernard A.P. Lafont and Jack R. Bennink, 2020; Published by Mary Ann Liebert, Inc. This Open Access article is distributed under the terms of the Creative Commons Attribution Noncommercial License (http://creativecommons.org/licenses/by-nc/4.0/) which permits any noncommercial use, distribution, and reproduction in any medium, provided the original author(s) and the source are cited. 
However, CD8+ T cells have been shown in animal models to definitively provide protection against IAV disease, and a beneficial role has been suggested in human studies $(10,11)$. The greatest benefit for $\mathrm{T}$ cell immunity has been argued for the case of novel pandemic IAV infections where preexisting B cell immunity is lacking.

On the contrary, a strain-matched B cell response can provide close to, if not, sterilizing protection. The limitation, as already stated, is the antigenic drift of the new strains that constantly arise in the global human population as immunity to the circulating strain increases in the population after infection or thanks to immunization with seasonal influenza vaccines. A few years ago, it was found that broadly crossreactive antibodies could be generated against IAVs, and inducing this type of immunity through vaccination is the focus of current enhanced efforts $(9,15,26)$. New vaccination strategies targeting the conserved domain of the HA stem are currently the favored approaches [for review, see Krammer (16)].

Significant gaps remain in our understanding of the tug of war between the host and the virus and the extent to which IAV evolves in response to host defense mechanisms. This information has critical implications for the development of universal vaccination strategies. For years, the dogma had been that neutralizing anti-HA antibodies provided all the pressure necessary for the antigenic changes to occur and for the virus to escape pre-existing immunity. Monoclonal antibodies and polyclonal sera have been shown to select influenza virus mutants dissimilarly in model systems $(5,30,33)$. While viral selection using a single monoclonal antibody may lead to HA point mutations in the antibody binding site, mutants arising under selection from polyclonal sera or combination of monoclonal antibodies exhibit increased avidity for cellular glycans and harbor mutations near the sialic acid binding site, distinct from the antibody binding sites (12,34). Similarly, studies investigating intrahost evolution of IAVs in vaccinated and nonvaccinated hosts (dogs, horses, and humans) have been unable to demonstrate selection of viral escape variants $(6,13,21,22)$. These studies suggest that immune-driven variants do not usually arise in immunocompetent individuals. This paradigm needs to be reconciled with the well-known IAV antigenic evolution observed at the population level in humans. Studies are needed to further our understanding of the mechanisms of population-based antigenic evolution and to evaluate the potential contribution from specific immune response (B cells and $\mathrm{T}$ cells) in driving such changes.

The objective of universal influenza HA vaccines is to generate a specific immunity in the human population to conserved regions that are broadly found in IAVs. Current candidate vaccine approaches are focused on the viral HA, particularly to the HA stem. To the extent that specific immunity can drive point mutations during an acute infection, a focused universal vaccine would create a prime opportunity for IAV to be pressured quickly and to evolve in that region. Therefore, it is important to consider what factors reduce the risk of rapid virus evolution and the mutations of antibody binding site or $\mathrm{T}$ cell epitopes. A more comprehensive look at virus evolution can be found in the review from Bloom and colleagues (31).

First, influenza is an acute and limited infection in immunocompetent individuals. Other natural virus infections where immune-specific mutations have been observed involve chronic infection with various viruses such as the human immunodeficiency virus (HIV), lymphocytic choriomeningitis, hepatitis, or herpes viruses $(3,4,14,18-20$, $25,28)$. In these infections, the virus is not cleared rapidly by host immunity, as in acute infections, and frequently persists in the host for weeks or years. Chronic infections provide sufficient time for mutant viruses less efficiently or not at all recognized by the immune system to outgrow the original virus and eventually to be transmitted. During acute infections, the time frame for virus evolution and transmission to the next host is compressed to a few days, making the process very unlikely in most cases. Thus, this kinetics issue provides one potential explanation why virus evolution is not typically observed for acute infections. In contrast, in immunocompromised individuals, influenza infection becomes more protracted, lasts for weeks, and is associated with frequent mutations in multiple viral genes (32).

A second explanation involves the inherent nature of immunity to viruses and proteins. Immune responses are complex involving repertoires of both $\mathrm{B}$ cells and $\mathrm{T}$ cells with multiple specificities and diverse immunodominance. For the virus, the most effective way to escape these responses is to make the minimal change that would affect the most specificities. One example of this process is the addition or shifting of glycosylation sites that have a broader structural impact than the single nucleotide change would normally predict $(1,36)$. Conversely for the host, targeting multiple viral epitopes or proteins complicates the virus' capacity to evade the response by forcing it to mutate multiple amino acids to avoid immune recognition during the limited time frame provided by acute infection. If very high concentrations of antibodies or high numbers of T cells of monoclonal specificity are pre-existing in the host at the time of infection, immune pressure could lead to selective virus evolution, even in an acute infection. However, the levels of monoclonal-specific response required for this process are highly unlikely to be present in a normal host. With each additional specificity, viral escape becomes progressively more difficult as the probability of the virus possessing multiple amino acid mutations is increasingly less likely and would require extremely high virus titers. Therefore, broadening the anti-HA specificities within an immune response should reduce the opportunity for point mutation evolution.

The effectiveness of the immune mechanisms related to each specific response (for B and T cells) is also important for the multiple specificities. For example, a specific neutralizing antibody capable of blocking infection or generating "sterilizing immunity" would likely be more effective than a specific cytotoxic $\mathrm{T}$ cells response that requires infection or a specific antibody that only works through antibody-dependent cellular cytotoxicity. The more effective the multiple individual immune response components are, the less likely specific adaptive mutations will occur in an individual. Conversely, the less effective the individual responses are, the more likely specific adaptive mutants could arise.

Finally, virus characteristics also contribute to limit the effect of immune-induced selection of specificity-selected variants. Influenza infection in the airways results in frequent superinfection of susceptible cells by multiple virus 
particles. This is the basis for the generation of reassortant viruses frequently detected for instance in zoonotic infection. This mechanism could also contribute to hinder the selection of mutant viruses, as unmutated antigens are still expressed and recognized by existing immune specificities during local coinfection, slowing down the kinetics of novel specificity selections. Furthermore, the segmented nature of the influenza virus genome complicates the selection of multiple mutation variants. While the generation of quasispecies virus is well known during influenza virus replication, the selection of multi-epitopic variants on different gene segments will probably proceed stepwise rather than synchronously as the mutations in different proteins will not be linked to one unique RNA strand.

So how can virus evolution be slowed? Perhaps clues may be gathered from chronic infections such as HIV or hepatitis $\mathrm{C}$ virus. In these infections, the virus can escape immune pressure mediated by both $\mathrm{T}$ cell and $\mathrm{B}$ cell specificities, leading to the generation of quasi-species in the host. The basis of virus evolution is the intrinsic unfaithful nature of the viral polymerase whose activity introduces systematically "errors" in the newly produced viral genomes and generates a pool of virus variants for selection, a concept shared by influenza viruses. For HIV, preventing viral replication using combinations of highly specific and synergetic drugs, called highly active antiretroviral therapies, is now the basis of viral suppression and has changed the lives of HIV-infected patients worldwide (17). These highly active antiretroviral therapies depend on the use of the multiple antiviral drugs to effectively suppress viral evolution and prevent the generation of drug-resistant viruses. Monotherapies have all failed to prevent HIV evolution and have resulted in the selection of drug-resistant viruses, a fate that is also true for the treatment of influenza with adamantanes.

In a similar manner, a host response comprising multiple synergistic specificities could help minimize specificityinduced virus evolution. This requires addition of supplemental target antigens to current approaches designed to generate broadly reactive specificities using minimally focused immunogens. For influenza, this could be achieved in multiple ways. In addition to targeting the conserved HA stem region, the goal might be to continue to immunize with HA to generate an updated broader response targeting the head. Recent studies have indeed suggested that HA head immunodominance over stem epitopes can be overcome by partitioning head from stem (2). Therefore, the additional specificities may have to be given in a separate immunization. Other target IAV proteins, such as the NA or the more conserved nucleoprotein or matrix, could also be considered to add breadth to the response. Any of these antigens would effectively broaden the vaccine response and reduce the opportunity for specificity-based virus evolution. To the extent possible, inducing responses associated with optimal effector function mechanisms, especially neutralization or preventing cell infection, would be ideal.

\section{Epilogue from J.R.B.}

It was a genuine privilege to be one of Peter's earliest graduate students. It was an extremely exciting time immediately after his Nobel winning discovery when he came to Philadelphia to work at the Wistar Institute and University of
Pennsylvania. Peter's curiosity and passion to understand the biology of T cell responses were infectious and inspirational. It was a pure pleasure to work and learn side by side with him at the bench. I have immense gratitude for the training he gave me and for all the support he has provided throughout the years since. Although this journal issue is for Peter, personally I would feel remiss if I did not mention his supporting foundation at home. I thank Penny for her kindness, grace, and generosity when we were graduate students but also over the years. I recognize and deeply appreciate her contributions to Peter's success and support to allow him to share his knowledge and insight to me as a trainee but also to the rest of the world.

\section{Disclaimer}

The opinions expressed in this article are the authors' own and do not reflect the view of the National Institutes of Health, the Department of Health and Human Services, or the United States government.

\section{Author Disclosure Statement}

No competing financial interests exist.

\section{Funding Information}

The authors are supported by the Intramural Research Program of NIH, NIAID, Bethesda, MD.

\section{References}

1. Altman MO, Angel M, Kosik I, et al. Human influenza A virus hemagglutinin glycan evolution follows a temporal pattern to a glycan limit. MBio 2019;10:pii:e00204-19.

2. Angeletti D, Kosik I, Santos JJS, et al. Outflanking immunodominance to target subdominant broadly neutralizing epitopes. Proc Natl Acad Sci U S A 2019;116:1347413479.

3. Borrow P, and Shaw GM. Cytotoxic T-lymphocyte escape viral variant show important are they in viral evasion of immune clearance in vivo? Immunol Rev 1998;64:37-51.

4. Bowen DG, and Walker CM. Mutational escape from $\mathrm{CD} 8^{+}$ T cell immunity: HCV evolution, from chimpanzees to man. J Exp Med 2005;201:1709-1714.

5. Davis AKF, McCormick K, Gumina ME, et al. Sera from individuals with narrowly focused influenza virus antibodies rapidly select viral escape mutations in ovo. J Virol 2018;92:pii: e00859-18.

6. Dinis JM, Florek NW, Fatola OO, et al. Deep sequencing reveals potential antigenic variants at low frequencies in influenza A virus-infected humans. J Virol 2016;90:3355-3365.

7. Effros RB, Doherty PC, Gerhard W, et al. Generation of both cross-reactive and virus-specific T-cell populations after immunization with serologically distinct influenza A viruses. J Exp Med 1977;145:557-568.

8. Ehrlich P. Nobel Lecture, December 11, 1908. Nobel Lectures, Physiology or Medicine, 1901-1921. Amsterdam: Elsevier Publishing Company, 1967.

9. Ekiert DC, and Wilson IA. Broadly neutralizing antibodies against influenza virus and prospects for universal therapies. Curr Opin Virol 2012;2:134-141.

10. Epstein SL, and Price GE. Cross-protective immunity to influenza A viruses. Expert Rev Vaccines 2010;9:13251341. 
11. Epstein SL. Prior H1N1 influenza infection and susceptibility of Cleveland Family Study participants during the H2N2 pandemic of 1957: an experiment of nature. J Infect Dis 2006;193:49-53.

12. Hensley SE, Das SR, Bailey AL, et al. Hemagglutinin receptor binding avidity drives influenza A virus antigenic drift. Science 2009;326:734-736.

13. Hoelzer K, Murcia PR, Baillie GJ, et al. Intrahost evolutionary dynamics of canine influenza virus in naïve and partially immune dogs. J Virol 2010;84:5329-5335.

14. Koenig S, Conley AJ, Brewah YA, et al. Transfer of HIV1-specific cytotoxic $\mathrm{T}$ lymphocytes to an AIDS patient leads to selection for mutant HIV variants and subsequent disease progression. Nat Med 1995;1:330-336.

15. Krammer F, and Palese P. Advances in the development of influenza virus vaccines. Nat Rev Drug Discov 2015;14: $167-182$.

16. Krammer F. Novel universal influenza virus vaccine approaches. Curr Opin Virol 2016;17:95-103.

17. Laskey SB, and Siliciano RF. A mechanistic theory to explain the efficacy of antiretroviral therapy. Nat Rev Microbiol 2014;2:772-780.

18. Lewicki HA, Von Herrath MG, Evans CF, et al. CTL escape viral variants. II. Biologic activity in vivo. Virol 1995; 211:443-450.

19. McMichael AJ. T cell responses and viral escape. Cell 1998;93:673-676.

20. Moskophidis D, and Zinkernagel RM. Immunobiology of cytotoxic T-cell resistant virus variants studies on lymphocytic choriomeningitis virus (LCMV). Semin Virol 1996;7:3-11.

21. Murcia PR, Baillie GJ, Daly J, et al. Intra- and interhost evolutionary dynamics of equine influenza virus. J Virol 2010;84:6943-6954.

22. Murcia PR, Baillie GJ, Stack JC, et al. Evolution of equine influenza virus in vaccinated horses. J Virol 2013;87:47684771.

23. Ngono AE, and Shresta S. Immune response to dengue and Zika. Annu Rev Immunol 2018;36:279-308.

24. Parker L, Wharton SA, Martin SR, et al. Effects of eggadaptation on receptor-binding and antigenic properties of recent influenza A (H3N2) vaccine viruses. J Gen Virol 2016;97:1333-1344.

25. Pewe L, Wu GL, Barnett EM, et al. Cytotoxic T cellresistant variants are selected in a virus-induced demyelinating disease. Immunity 1996;5:253-262.

26. Pica N, and Palese P. Toward a universal influenza virus vaccine: prospects and challenges. Annu Rev Med 2013;64: 189-202.

27. United States of America, Presidential Executive Order on Modernizing Influenza Vaccines in the United States to
Promote National Security and Public Health, September 19, 2019. www.whitehouse.gov/presidential-actions/execu tive-order-modernizing-influenza-vaccines-united-states-pro mote-national-security-public-health. (accessed October 24, 2019).

28. Vanderford TH, Bleckwehl C, Engram JC, et al. Viral CTL Escape mutants are generated in lymph nodes and subsequently become fixed in plasma and rectal mucosa during acute SIV infection of macaques. PLoS Pathog 2011;7: e1002048.

29. von Behring E, and Kitasato S. Ueber das zutandekommen der diphtherie-immunitat und der tetanus-immunitat bei thieren. Deutsche Medizinsche Wochenschrift [German]. On the establishment of Diphtheria immunity and Tetanus immunity in animals. German Medical Weekly. 1890;16:1113-1114.

30. Webster R, and Laver W. Determination of the number of nonoverlapping antigenic areas on Hong Kong (H3N2) influenza virus hemagglutinin with monoclonal antibodies and the selection of variants with potential epidemiological significance. Virology 1980;104:139-148.

31. Xue KS, Moncla LH, Bedford T, et al. Within-host evolution of human influenza virus. Trends Microbiol 2018;26: 781-793.

32. Xue KS, Stevens-Ayers T, Campbell AP, et al. Parallel evolution of influenza across multiple spatiotemporal scales. Elife 2017;6:e26875.

33. Yewdell J, Webster R, and Gerhard W. Antigenic variation in three distinct determinants of an influenza type A haemagglutinin molecule. Nature 1979;279:246-248.

34. Yewdell JW, Caton AJ, and Gerhard W. Selection of influenza A virus adsorptive mutants by growth in the presence of a mixture of monoclonal antihemagglutinin antibodies. J Virol 1986;57:623-628.

35. Zinkernagel RM, and Doherty PC. Restriction of in vitro $\mathrm{T}$ cell-mediated cytotoxicity in lymphocytic choriomeningitis within a syngeneic or semiallogeneic system. Nature 1974; 248:701-702.

36. Zost SJ, Parkhouse K, Gumina ME, et al. Contemporary $\mathrm{H} 3 \mathrm{~N} 2$ influenza viruses have a glycosylation site that alters binding of antibodies elicited by egg-adapted vaccine strains. Proc Natl Acad Sci U S A 2017;114:12578-12583.

Address correspondence to:

Dr. Jack R. Bennink

Viral Immunology Section

NIAID

$\mathrm{NIH}$

Bethesda, MD 20892-8001

E-mail: jbennink@nih.gov 